\title{
La producción de narrativas como herramienta de investigación y acción sobre el dispositivo de sexo/género: Construyendo nuevos relatos
}

Narrative production as an action-research tool on the sex/gender dispositive: Constructing new stories

\author{
Antar Martínez-Guzmán \\ Universidad de Colima \\ Marisela Montenegro \\ Universitat Autònoma de Barcelona
}

Resumen

Los abordajes narrativos muestran una creciente presencia en las ciencias sociales y con frecuencia se argumenta su utilidad para el estudio de la identidad. En este trabajo pretendemos discutir algunas las implicaciones de los abordajes narrativos para el estudio de las identidades sexo-genéricas. En un primer momento, mostramos algunas las diferencias relevantes que existen la aproximación narrativa y otras formas de análisis del discurso. Posteriormente, discutimos las potencialidades de la perspectiva narrativa para el estudio del sexo-género como dispositivo semiótico-material. Finalmente, elaboramos una propuesta particular de comprensión y desarrollo de abordaje narrativo en torno a las identidades sexogenéricas, donde los relatos o narraciones no son sólo instrumentos de producción de conocimientos sino también vehículos para la acción social. A manera de ilustración, citamos dos experiencias de investigación utilizando la metodología de las producciones narrativas.

Palabras clave: Investigación narrativa; Género; Producciones narrativas; Acción social

\section{Abstract}

Narrative approaches show a growing presence in the social sciences and are frequently understood to be useful for the study of identity. In this paper, we discuss some implications of narrative approaches to the study of sex/gender identities. First, we show some relevant differences between the narrative approach and other forms of discourse analysis. Subsequently, we discuss the narrative perspective potentiality for the study of sex and gender as material-semiotic dispositive. Finally, we developed a particular way of understanding and developing narrative research about sex/gender identities, where stories and narratives are not only means for knowledge production but also for social action. To illustrate this, we cite two research experiences using narrative production methodology.

Keywords: Narrative research; Gender; Narrative productions; Social action 


\section{Introducción}

El mundo está colmado de narraciones y relatos. Las narraciones, los relatos y las historias son formas ubicuas de comprender y organizar la vida social. Constituyen modalidades cotidianas y espontáneas con que damos cuenta y organizamos los mundos que habitamos. Las historias tejen relaciones sociales y, al mismo tiempo, se distribuyen a través de ellas. Acaso es ésta la persistencia evocada por Roland Barthes (1977, p. 67) cuando advierte que la narrativa "simplemente está ahí, como la vida misma".

Así, las narrativas -como formas discursivas que interconectan acontecimientos y entrelazan diferentes actores (sujetos, objetos) entre sí de una forma particular- juegan un papel relevante a la hora de constituir y movilizar las identidades que construimos (Bruner, 1991; Gergen, 2007). Esta constatación ha permitido que los abordajes narrativos muestren una creciente presencia en las ciencias sociales y que con frecuencia se argumente sobre su utilidad para el estudio de los procesos identitarios (Ricoeur, 2000; Riessman, 2008).

Hasta el llamado "giro discursivo" (Ankersmit, 2001; Rorty, 1998) y el propio "giro narrativo" (Kreiswirth, 1992) en las ciencias sociales, los estudios narrativos estuvieron confinados principalmente a los ámbitos de la lingüística y la crítica literaria. Estos "giros" sientan las bases para desdibujar las fronteras entre distintas de disciplinas y transpolar la perspectiva narrativa al estudio de diversas cuestiones sociales y humanas hasta convertirle en un fecundo campo interdisciplinario (Pinnegar y Daynes, 2007; Polkinghorne, 1988).

En el ámbito de las ciencias sociales y, particularmente, en la investigación psicosocial, es común que las preguntas que hacen las investigadoras sean respondidas por las participantes en forma de relatos o extensas tramas que aparentemente se apartan de la pregunta. De forma espontánea, el sujeto tiende a responder a la interpelación a través historias y a generar sentido a través de relatos. Es posible -de hecho, ha sido frecuente- que quien investiga interprete estas respuestas como digresiones o "ruido" en torno a las preguntas concretas que ha formulado. De esta manera, las respuestas en forma de relato a menudo desafían la lógica analítica de la investigación psicosocial al uso; una lógica erigida en la identificación de categorías, por ejemplo (Riessman, 2002). Sin embargo, los factores y elementos que juegan papeles determinantes en los procesos estudiados a menudo sólo adquieren sentido al interior de las tramas narrativas en que están insertos. El reciente auge de las metodologías narrativas responde, en parte, a este intento por reconocer y recoger las modalidades espontáneas en que los sujetos producen sentido y se conducen en su vida cotidiana.

Recientemente se han desarrollado fértiles líneas de abordaje narrativo en el estudio de las relaciones de género y de las diferencias sexuales. Estas líneas proveen no sólo de dispositivos analíticos en el plano metodológico, sino de distintas coordenadas teóricas para comprender la relación entre subjetividad, lenguaje y acción en las experiencias sexogenéricas. Así, el modo narrativo propone un particular modo de ser, de conocer y de hacer, articulando tres registros en un abordaje específico: el ontológico, el epistemológico y el metodológico.

En el contexto de esta progresiva propagación de los abordajes narrativos, este trabajo pretende discutir algunas de sus implicaciones para el estudio de las identidades sexogenéricas. La discusión se focaliza predominantemente en los aspectos teóricometodológicos de los abordajes narrativos, en el contexto de la investigación cualitativa y crítica, implicados en la comprensión de los procesos identitarios en el ámbito de la sexualidad y el género, acentuando la constitución y la apropiación de sexualidades abyectas e identidades no normativas.

En un primer momento, se discuten algunas diferencias relevantes que se identifican entre los abordajes discursivos y la aproximación narrativa, haciendo énfasis en las particularidades de ésta última en contraste con otras formas de "análisis del discurso". Posteriormente, se discuten las potencialidades de la perspectiva narrativa para el estudio del sexo/género como dispositivo semióticomaterial y, particularmente, para el análisis de las identidades sexo-generizadas. Se enfatizan especialmente aquellas formas de aproximación narrativa que evidencian los entramados políticos naturalizados y que a la vez reconocen y dan cuenta de la agencia de los sujetos que narran. 
A continuación se presenta una propuesta particular de comprensión y desarrollo de abordaje narrativo en torno a las identidades sexo-genéricas, donde los relatos o narraciones no son sólo instrumentos de producción de conocimientos sino también vehículos para la acción social. Este particular abordaje gira en torno a la co-producción de narrativas (Balasch y Montenegro, 2003) como método de investigación y herramienta para la acción en el contexto de las disputas en torno a los "regímenes de verdad" (Foucault, 1988/1990) y a las condiciones de posibilidad de diversas expresiones/identidades sexo-genéricas.

A manera de ilustración de este abordaje, se intercalan algunos fragmentos de narrativas elaboradas en dos experiencias de investigación en torno a identidades de género nonormativas utilizando la metodología mencionada. A modo de cierre, se señalan algunas de las posibilidades y fortalezas de esta aproximación narrativa, enfatizando la importancia metodológica y política de la construcción de nuevos relatos en el ámbito del género.

\section{Narrativa y discurso}

Iniciemos situando a los abordajes narrativos como parte de $(y$, por tanto compartiendo orígenes con) la amplia galaxia de los estudios del discurso (van Dijk, 1997). Si bien este punto de partida podría ser controvertido argumentando que el "giro narrativo" es suficientemente amplio y contiene dentro de sí sus propias concepciones teóricometodológicas de forma que puede ser considerado campo aparte, lo cierto es que comparte con el espectro de los estudios del discurso un tronco común que indudablemente les emparenta.

Entre las raíces comunes encontramos fundamentalmente un cambio radical en la concepción del lenguaje y su función en la vida social. El giro en la concepción del lenguaje plantea una perspectiva donde: a) el lenguaje no se concibe como un sistema abstracto de signos y símbolos de carácter formal, sino como práctica social cotidiana y dinámica ejecutada continuamente por los sujetos parlantes; b) el lenguaje no es un medio meramente descriptivo de la realidad, sino que tiene propiedades constructivas y constitutivas con respecto a la misma; y c) el lenguaje en uso o el discurso están irremediablemente signados por sexo, clase, raza, cultura y otras marcas de las posiciones desde donde se enuncian (Íñiguez-Rueda, 2003; Wodak, 2003).

Con este telón de fondo, cuando hablamos de relatos o historias bien podemos estar hablando de "discursos narrativos". Las historias y los relatos son concebidos como narrativas que a su vez son textos $y$, por tanto, discursos. De hecho, en su concepción más amplia, un relato puede definirse, según David Boje (1991), como cualquier dispositivo discursivo que genera y sostiene significado, por lo que cualquier texto con significado puede en última instancia comprenderse como una narrativa. Con todo, las aproximaciones narrativas han desarrollado modos peculiares de estudio y concepción del lenguaje que les diferencian de otras formas de análisis del discurso. Así, puede hablarse de una especificidad del abordaje narrativo en el contexto del vasto ámbito de los estudios del discurso.

¿En qué consiste dicha especificidad? Sin afán de hacer un análisis pormenorizado de las heterogéneas y a menudo debatidas características de la aproximación narrativa, delineamos algunos elementos clave para identificar los aportes particulares que pueden ofrecer los abordajes narrativos para la comprensión de la subjetividad, la identidad y, más concretamente, de las identidades sexogenéricas.

A grandes rasgos, la narrativa puede entenderse como un texto -escrito o hablado-que involucra una trama donde diferentes acontecimientos y actores son interconectados (Clegg y Bailey, 2008). Las narrativas implican cadenas temporales de acontecimientos o acciones interrelacionados, llevadas a cabo por personajes. No son simples instantáneas fotográficas, sino que requieren secuenciación y argumento (Bruner, 1986; Gergen, 2007). Al narrar, la persona busca darle forma al espacio y al tiempo, generar dispositivos de cohesión que le permiten hilar la acción; las narrativas crean tramas y dramas y, al hacerlo, generan sentido sobre la experiencia de las narradores, de las situaciones sociales y de la historia (Crossley, 2000).

Esta función de entramado, interconexión, asociación y vinculación de elementos en una totalidad singular confiere a las aproximaciones narrativas un primer elemento distintivo: lo que emerge a un primer plano de estudio 
no son tanto los acontecimientos, los actores o los elementos aislados, sino el ordenamiento, la organización y la particular articulación de estos elementos que la narrativa plantea. Así, y en contraste con otras aproximaciones de análisis del discurso caracterizadas por diseccionar el texto y centrar el análisis en elementos separados y específicos (e.g. el análisis de repertorios interpretativos propuesto por Jonathan Potter y Margaret Wetherell, 1996), el abordaje narrativo se interesa en primer término por interrogar la trama y el particular ensamblaje de eventos y personajes consumado en la narración. El estudio de narrativas toma como principal unidad de interés a la historia en sí misma.

Por supuesto, los elementos del relato juegan un papel importante a la hora de interrogar la narración, pero éstos son considerados principalmente en función del rol que juegan en el entramado intertextual generado en la trama. El análisis narrativo no sólo nos informa sobre el contenido de un texto sino también sobre la forma en que se cuenta la experiencia: no se centra solamente en el contenido al que el lenguaje se refiere sino también en la manera en que la historia es contada (Clough, 2002).

Por otra parte, la aproximación narrativa reconoce y otorga un papel relevante al sujeto que narra. Se interesa por el modo en que un sujeto o actor social cuenta algo sobre su propia vida o experiencia. Así, la elaboración de un relato es el logro de la actividad narrativa del sujeto. En el relato se integran los aspectos "encarnados" de una explicación, se incorporan en un punto de vista diferentes aspectos de la vivencia del individuo con respecto a un tema.

A diferencia de otras aproximaciones discursivas, donde el sujeto se diluye y el lenguaje adquiere el papel de actor protagonista, el abordaje narrativo concede a la narradora un rol activo y constructivo; el sujeto genera una narración particular, propia de su posición, perspectiva y subjetividad. Así, tanto la narración como el sujeto entran de forma relevante dentro del campo de interés. Por supuesto, el sujeto no puede elaborar cualquier relato, pues está constreñido por un conjunto de discursos normativos, narrativas hegemónicas y recursos simbólicos disponibles en determinado espacio social (Gergen, 2007). Empero, aún a partir de estos recursos limitados y márgenes previos, elabora un ordenamiento específico y un relato singular.

En este sentido $-\mathrm{y}$ aquí se encuentra otro de sus rasgos característicos- la interrogación narrativa permite acceder simultáneamente tanto al imaginario cultural y social más amplio en que el sujeto se encuentra inmerso, como a la perspectiva propia de la persona que cuenta el relato. El abordaje narrativo busca dar cuenta de cómo se articulan la dimensión biográfica, la histórica y la social. Una narrativa nos puede informar, a través de su particularidad, sobre los valores, las normas, las convenciones y otras fuerzas sociales y culturales presentes. Las perspectivas de los sujetos están siempre localizadas en tiempos y espacios particulares. Las cuestiones y problemáticas que las personas refieren en sus relatos provienen al mismo tiempo de la experiencia personal como de la sociedad en que viven (Riessman, 2002). Así, el trabajo con narrativas nos ofrece un abordaje donde se desdibujan las dicotomías 'personal-social' y 'micro-macro': la idea consiste en aproximarse a la singularidad de una narrativa entendiendo esta singularidad como la manera en que las fuerzas sociales se intersectan en las trayectorias individuales.

Otro rasgo definitorio de la perspectiva narrativa se refiere a su potencialidad para mostrar el carácter contextual de los discursos elaborados por diferentes actores sociales. Las narraciones desarrollan los acontecimientos y las explicaciones a partir de una fuerte carga indexical, en el sentido de que hacen constante referencia a espacios, tiempos y actores situados en contextos particulares donde la acción se lleva a cabo. Los relatos son ricos en enunciaciones indexicales puesto que a) se erigen en torno a la experiencia personal, y b) tienden a ofrecer detalles sobre acontecimientos y acciones localizados. Esta función permite una indagación donde se privilegia el carácter situado y encarnado de los relatos producidos.

De acuerdo con Marin Bauer (1996), la producción de un relato sigue un curso dirigido por tres rutas: a) textura detallada, donde se ofrece información prolija y suficiente para hacer plausible la interconexión entre diferentes acontecimientos o la transición entre un evento y otro; b) fijación de la relevancia, donde la narradora da cuenta de aquellas características de los acontecimientos que son 
relevantes de acuerdo con su particular visión del mundo, es decir, el relato de los acontecimientos es selectivo; y c) cierre de gestalt, las historias son articuladas como unidades completas (e.g. con inicio, desarrollo y desenlace) y con sentido propio. Esta organización tripartita hace que el relato fluya una vez que ha iniciado y contribuye a generar la organización global característica del discurso narrativo. Así pues, la actividad narrativa continuamente reproduce el espectro de un todo, de un sistema articulado, donde inclusive, como ha hecho notar Judith Roof (1996), la propia idea de que hay tales cosas como 'partes' y 'todos' es ya un efecto de la organización narrativa.

Estos rasgos particulares propios de la aproximación narrativa permiten comprender la narrativa de forma simultánea como objeto de estudio, método de indagación y producto de investigación. De igual manera, muestran que la narrativa puede abordarse a la vez como proceso y como fenómeno. Como proceso o método resulta en una lógica de indagación; como fenómeno representa la estructura cualitativa de la experiencia (Clandinin y Connelly, 1994).

En tanto sentido de orden de los acontecimientos y proceso a través del cual personajes, causas y efectos se combinan en patrones percibidos como coherentes, la narrativa es la lógica generadora a partir de la cual la individualidad, la identidad y la ideología se fusionan en una visión unificada de la experiencia (Roof, 1996). De hecho, para algunas investigadoras narrativas, la historia es la unidad fundamental que da cuenta de la experiencia humana (Pinnegar y Daynes 2007) y, en consecuencia, puede resultar también el canal idóneo para explorarla. Así, las narrativas participan de una lógica recursiva donde juegan el papel de principios organizadores al mismo tiempo que son estructuradas a partir de los imaginarios culturales y los discursos dominantes.

Los elementos mencionados pueden ser útiles para distinguir la sensibilidad narrativa de otras formas de indagación cualitativa y discursiva. Hay que advertir, sin embargo, que en muchos casos las fronteras entre diferentes abordajes teórico-metodológicos son difusas y por momentos pierden sentido. Además, es importante reconocer que las perspectivas narrativas componen un terreno amplio que abarca una gama de métodos biográficos, de indagación de la experiencia personal y de estudios discursivos/literarios que quedan fuera del escrutinio detallado en este somero panorama. Con todo, es posible bosquejar un perfil provisional de la sensibilidad narrativa donde se muestren especialmente algunos de los recursos que ofrece para la investigación psicosocial y, particularmente, para la comprensión del género.

\section{Narrativa y género}

Los vínculos posibles entre las perspectivas narrativas de investigación y los estudios de género son múltiples y polivalentes. Sin afán de hacer una revisión exhaustiva, resulta conveniente rescatar algunas potencialidades del abordaje narrativo como herramienta para el análisis del dispositivo de sexo/género ${ }^{1}$ y de las identidades sexo-genéricas, enfatizando especialmente en aquellos recursos de la mirada narrativa que pueden contribuir a visibilizar los entramados políticos naturalizados con respecto al sexo-género y a reconocer la agencia de los sujetos que narran.

Para explorar la (a menudo tensa) relación entre perspectivas narrativas y estudios de género podemos evocar la pregunta que, desde las coordenadas de los estudios literarios feministas, plantea Susan Lanser (1986): ¿sobre qué cuerpo de textos, sobre qué comprensiones de la narrativa y qué universo referencial, las indagaciones de la narratología se han fundado? La pregunta de Lanser vislumbra ya una línea de cuestionamientos que el feminismo y las perspectivas críticas plantearán sobre el género haciendo uso de los abordajes narrativos: ¿cuáles son las historias posibles en contextos determinados?, ¿qué cuerpos e identidades habitan estas historias o son exiliados de las mismas?, ¿con qué términos o desde qué perspectivas las historias dominantes se articulan?, ¿en qué medida contribuyen a (re)producir diferencias sexogenéricas y relaciones de dominación a partir de ellas?

Hasta hace algunas décadas, diversas voces feministas denunciaban la virtual inexistencia

\footnotetext{
1 'Dispositivo' en tanto red de elementos heterogéneos que actúa estratégicamente para regular ciertas relaciones sociales, y de 'sexo-género', expresión compuesta que busca trascender la dualidad naturaleza-cultura y enfatizar la imbricación de ambos términos para comprender las relaciones de poder en dicho ámbito.
} 
de trabajos en el campo de los estudios narrativos que tomaran en cuenta la forma en que las fuerzas de género atravesaban en múltiples dimensiones los relatos estudiados. Así, la indagación narrativa se venía realizando predominantemente, en palabras de Lanser (1986, pp. 199), "sobre textos masculinos o sobre textos tratados como textos masculinos". La narración masculina (heterosexual, blanca, occidental...) se erige entonces como la narración universal.

Pronto se desarrollarán líneas de crítica e indagación que, alimentadas por perspectivas feministas y queer (Freeman, 2010), pondrán de manifiesto que las narrativas no existen y no pueden operar con independencia de otros modos -en buena medida vinculados a la identidad, el género y la sexualidad- a través de los cuales organizamos nuestra experiencia. Así, muestran que las categorías sexogenéricas son en cierta forma categorías narrativas, tanto en nuestra comprensión de las mismas como en la forma en que prefiguran y atraviesan determinadas formas narrativas (Roof, 1996).

En mitad de esta tensión, el vínculo entre la investigación narrativa y el género puede describirse, de forma muy esquemática, a partir de una relación de doble sentido: a) la exploración de las formas (contingentes e históricas) a través de las cuales el sexo y el género son modelados por medio de las narrativas socialmente disponibles; y b) la forma en que los presupuestos dominantes de sexo/género informan y articulan las narrativas a través de las cuales se constituyen diversas relaciones sociales y comprensiones del mundo (Lanser, 2013). Esta relación recursiva - de mutua determinación entre relatos y políticas sexogenéricas- hace de la relación entre narrativa y género un vínculo a la vez problemático y fecundo; un campo de problematización y al mismo tiempo de intervención en los mecanismos de regulación política de los placeres, los cuerpos y las identidades.

En el contexto de esta relación recursiva, es posible delinear tres líneas teóricometodológicas en que la perspectiva narrativa puede contribuir a enriquecer la indagación crítica sobre el género y la sexualidad. En primer lugar, la perspectiva narrativa permite poner en primer plano la subjetividad presente en el proceso de producción de conocimiento $\mathrm{y}$, en este sentido, los vectores políti- cos que se ponen en juego. En contraste con un modelo de ciencia donde la pretensión de objetividad oculta las relaciones de poder y genera una mirada colonizadora sobre los sujetos a los que se refiere (Harding, 1996), la indagación narrativa privilegia la posicionalidad y la subjetividad de toda enunciación y todo relato.

En este sentido, la indagación narrativa se vuelve una indagación política: ¿quién cuenta la historia?, ¿desde dónde?, ¿a quién apela?, ¿qué versiones de los acontecimientos se ponen en juego? Del mismo modo, la producción de narraciones y relatos en el espacio social y político de las relaciones de género es una vía de entrada a un campo de batalla que implican decisiones concretas: ¿Qué palabras utilizo?, ¿qué personajes y sucesos quedan incluidos o excluidos?, ¿de qué manera organizo las relaciones entre los mismos?, ¿qué principios o finales confecciono?, ¿cómo describo la escena?

Así, la narrativa permite que la subjetividad suba a escena con un papel relevante, una subjetividad encarnada y situada en un contexto histórico y cultural específico. En la narración de un sujeto hay implicada una narración 'experienciada'. La narrativa no es cualquier explicación, es una explicación imbricada con una vivencia particular. Al mismo tiempo, en resonancia con las epistemologías feministas, la narrativa da lugar a un sujeto con agencia y creatividad; visibiliza la mediación del actor social; da cuenta del trabajo identitario que hace el sujeto y de la producción agenciada de su propio relato.

En segundo lugar, la perspectiva narrativa ofrece vías fértiles para abordar, a nivel metodológico, las preocupaciones de los feminismos post-estructuralistas y perspectivas queer sobre la forma en que el sujeto o el self se constituyen en el marco de discursos históricos y sociopolíticos. La articulación de la identidad de género, del cuerpo y del deseo se tornan espacios de problematización.

Al fundarse sobre la idea de que el discurso ostenta un papel realizativo y constructivo, la aproximación narrativa permite explorar la forma en que los sujetos - particularmente, el sujeto generizado- se constituyen y definen en el marco de discursos y narraciones. El sujeto generizado es, en este sentido, el resultado de procesos discursivos y performativos 
(Butler, 1999/2007). La perspectiva performativa caracteriza el género como el efecto de la actuación reiterada que produce la ilusión de un género estático o natural, mientras que oculta la inestabilidad y la contradicción propias de los actos de género de los sujetos.

La aproximación narrativa abre vías metodológicas para indagar en este proceso de producción de sujetos y subjetividades a partir del discurso. Así, por ejemplo, cuando contamos una historia sobre nuestras vidas estamos dando forma o performando la identidad que habitamos. Las narradoras, a través de sus relatos, no revelan un self esencial y nuclear sino que construyen a través de la propia actividad narradora un self parcial, dialógico y situado, a partir de los elementos discursivos y simbólicos disponibles. Las narrativas evidencian la forma en que los sujetos se posicionan en determinados espacios sociales y ante determinadas audiencias.

En este contexto, los relatos generados por actores sociales singulares y situados, por narradoras que habitan espacios no normativos o que generan rupturas con respecto al sistema sexo-genérico dominante, también realizan acciones y tienen un potencial performativo. Pueden ofrecer un medio para desafiar los discursos dominantes sobre el cuerpo, la sexualidad y el género. Como ha argumentado Catherine Riessman (2008), aproximarnos a la identidad (de género) como una lucha performativa sobre el significado de la experiencia abre posibilidades analíticas que están olvidados por las concepciones estáticas de la identidad y por las teorías esencialistas que asumen la unidad de un self interior, que además hacen juego con modelos sexogenéricos coercitivos y excluyentes.

En tercer lugar, es posible rescatar un replanteamiento que la aproximación narrativa permite en torno a la relación metodológica que establecen las participantes en una investigación. En este sentido, la narrativa facilita hacer evidente y explícito el proceso de coconstrucción de conocimiento que es propio no sólo de la narrativa sino de la investigación cualitativa en general: el abordaje narrativo permite reconocer el rol activo de la participante y mostrar la colaboración metodológica que se precisa para la producción de conocimiento.
De acuerdo con Amos Hatch y Richard Wisniewski (1995), una de las diferencias principales entre la narrativa y otros abordajes de investigación cualitativa reside en su naturaleza discursiva y dialógica, donde la narradora y la investigadora logran una comprensión mutua o intersubjetividad. El abordaje narrativo se desenvuelve en una relación tripartita entre narradora, oyente y narrativa (véase Blumenfeld-Jones, 1995). Su éxito radica en un acuerdo entre quien narra y quien escucha con respecto a cierto sentido compartido de la explicación o del relato en juego. Investigadora y participante entran en una relación en la que -se espera- ambas partes aprenderán y se transformarán en un encuentro dinámico. Esta relación entre investigadora y participante es, de acuerdo con Barbara Cole (2009), uno de los puntos fuertes de la perspectiva narrativa, puesto que incorpora y reconoce la "voz" propia de la participante.

En suma, la perspectiva narrativa es una herramienta útil para iluminar algunos aspectos complejos y a menudo velados sobre las relaciones de poder que constituyen las identidades generizadas $y$, al mismo tiempo, permite alejarse de aproximaciones reduccionistas y esencialistas (Cole, 2009). Por su singular registro metodológico y ontológico, la perspectiva narrativa permite un abordaje que enlaza -en consonancia con la agenda feminista y, posteriormente, la queer- lo personal y lo político, sobre la base de la experiencia "encarnada" y la visión situada.

El uso de las herramientas narrativas para el análisis crítico de las identidades y relaciones sexo-genéricas puede operar de múltiples maneras. Por ejemplo, a través de la socialización de historias alternativas y la indagación en la vida de individuos en posiciones de opresión, a través del desplazamiento de los análisis binarios para representar al sujeto como un otro múltiple (Peterson, 2006) o a través de la visibilización de relatos que pueden problematizar y complejizar nuestro entendimiento de la experiencia generizada y las múltiples fuerzas que la regulan. En cualquier caso, como mantiene Soyini Madison (1998), la perspectiva narrativa contribuye a crear espacios donde los sistemas dominantes y naturalizados de género son identificados $e$ interrogados. 


\section{Narrativa y acción}

El abordaje narrativo en torno a las identidades sexo-genéricas puede entenderse no sólo como un instrumento de producción de conocimientos sino también como un vehículo para la acción social. En este apartado proponemos delinear algunas formas en que podemos vincular narrativa y acción social. Para ilustrar someramente estos vínculos recurriremos a fragmentos de narrativas obtenidas en dos investigaciones donde se ha utilizado la metodología de las producciones narrativas para estudiar las identidades de género no normativas, particularmente las identidades transgénero ${ }^{2}$; una de estas investigaciones fue realizada en el contexto español y otra en el mexicano.

La propuesta metodológica de las producciones narrativas (Balasch y Montenegro, 2003) pone de relieve la producción de conocimiento como una actividad social y enfatiza aquellos aspectos del abordaje narrativo que pueden ser pensados como herramientas de investigación-acción. Esta metodología, fundamentada en la epistemología feminista de los conocimiento situados (Haraway, 1995), asume que todo conocimiento está marcado por determinadas coordenadas semióticomateriales de enunciación y por sus contextos de producción. Así, las producciones narrativas ofrecen un "aparato metodológico que opera promoviendo la localización de la mirada desde la cual se produce el conocimiento" (Balasch y Montenegro, 2003, p. 45). De igual forma, considera que las narrativas se producen en un contexto dialógico, donde quien enuncia participa de un proceso relacional activo y de una red de géneros de habla. Se asume, pues, que la narración es una práctica relacional donde se ponen en juego componentes discursivos plurales y múltiples.

Esta propuesta metodológica consiste en la producción conjunta de un "texto híbrido" entre investigadora y participante, que se genera a través de sesiones de conversación sobre el tema de estudio, la elaboración de texto -textualización- sobre dichas conversaciones

2 Sin afán de ofrecer una definición cerrada y definitiva, por 'transgénero' (o su apócope 'trans') entenderemos un conjunto amplio de manifestaciones identitarias que no se acoplan totalmente al binomio hombre-mujer tal cual está establecido por las normas de género dominantes. En este 'término paraguas' se agrupan posiciones diversas como la transexualidad y el travestismo. por parte de la investigadora, y la agencia de la participante sobre el texto para modificarlo y hacerlo concordar gradualmente con su punto de vista. A través de ciclos que incluyen la conversación, la textualización y la intervención de texto por parte de la participante, se concluye con una narración que muestra de manera expresa la visión de la participante sobre el tema de estudio, en este caso, sobre la propia identidad trans. Por tanto, no se recogen las palabras literales de la participante sino la forma en que ésta desea que se leída su perspectiva sobre el tema.

En este sentido, las autores advierten que las articulaciones posibilitadas por esta metodología:

Permitirán producir significados y fijaciones parciales de sentido del fenómeno, que se posicionarán en relación antagónica respecto a otros significados que operan en la comprensión del fenómeno. Por esta razón el conocimiento producido se orienta hacia la cuestión de responsabilidad política y no de la representación de una realidad externa (Balasch y Montenegro, 2003, p. 42).

Ciertamente, hay muchas formas en que puede decirse que narrativa y acción se imbrican, que narrar algo es hacer algo, esto es, llevar a cabo una acción. Aquí mostraremos algunas de ellas que se vuelven particularmente evidentes en el desarrollo de la metodología descrita. La primera de estas formas se refiere, como ya se ha mencionado, al carácter performativo de la narración. Las narrativas instauran verdades y marcos de comprensión específicos. Así, por ejemplo, las narrativas dominantes o meta-narrativas (Lyotard, 1984) funcionan como mecanismos donde historias particulares y parciales se posicionan como reflejos de la naturaleza. En el caso de las identidades transgénero, esto se hace evidente en los discursos médico-psiquiátricos que construyen estas expresiones identitarias como patológicas y, de esta manera, estigmatizan las experiencias y regulan un conjunto de condiciones prácticas y materiales en torno a las vidas transgénero (ver Martínez-Guzmán e Íñiguez-Rueda, 2010). Estos discursos producen cuerpos e identidades patologizadas, mientras se ofrecen como la única forma concebible o legítima de leer la experiencia sexogenérica.

Como argumenta Jean-François Lyotard (1984), debido a que la narrativa es el método comunitario a través del cual el conocimiento se almacena y se intercambia, éstas 
definen lo que tiene derecho a ser dicho y hecho en una cultura determinada $y$, puesto que son parte de esta cultura, están legitimadas por el sencillo hecho de que hacen lo que hacen. En este contexto, y partiendo de la premisa según la cual el lenguaje es una práctica social, las producciones narrativas pueden ofrecer vías metodológicas para conocer este entramado discursivo pero también para actuar sobre él.

La cuestión es que, al considerar solamente el nivel 'representacional' del lenguaje, se desaprovecha una oportunidad para generar estrategias 'activas', más reflexivas y críticas, para acometer la tarea de transformar los soportes discursivos que mantienen un curso particular de eventos, un arreglo social específico. Los relatos sobre género y sexualidad con que los actores sociales se entienden a sí mismos y son entendidos por otros actores no sólo funcionan como medios de comunicación de la experiencia o el conocimiento, sino que juegan un papel medular en el mapeo de las relaciones sociales y las disposiciones materiales de cierta economía de género. Por tanto, la transformación de las prácticas narrativas y discursivas sobre el género y la sexualidad es un punto crucial en la agenda sociopolítica de las identidades no normativas.

Otra forma en que podemos decir narración y acción se emparentan se observa en la manera en que las narrativas se desarrollan en una estructura orientada a la acción: se ofrece un contexto, los eventos se concatenan y terminan en un punto determinado; la narración incluye una especia de evaluación de los resultados (Bauer, 1996). Situación, establecimiento de objetivos, planificación y evaluación de los resultados son elementos constituyentes de las acciones sociales. La narración reconstruye tales acciones y sus contextos de una forma orgánica: revela lugares, tiempo, motivaciones y el sistema simbólico que orienta a los actores. Pongamos como ejemplo el fragmento de una narrativa construida con Carlos, un chico trans barcelonés:

A mí en lo personal no me resulta cómodo exponerme como transexual. Es complicado y difícil afrontar constantemente las posibles reacciones de la gente, las cosas que no llegan a comprender o que pueden causar incomodidad en la relación. Para muchas personas transexuales es muy importante conservar y proteger la intimidad de sus identidades, no ser constantemente leídos a través de ese filtro. En el mundo empresarial, por ejemplo, que es donde yo me muevo, si el DNI no coincide con la identidad en que vives es común que inmediatamente esta incongruencia genere desconfianza. $Y$ ese puede ser un factor que te dificulte el acceso al trabajo, a pesar de que puedas tender un currículum impresionante. Las personas necesitan credibilidad para poder desenvolverse en este entorno social. Necesitan proyectar una imagen que sea creíble y aceptable para los demás. Y esta credibilidad no es solo física, tiene que ver con el conjunto de la persona. Por ello, es muy importante buscar una imagen en que te reconozcas tú, en la cual te sientas bien contigo mismo, pero que también puedan reconocer los demás (Carlos, Barcelona, Narrativa, Febrero de 2009) ${ }^{3}$.

El relato de Carlos nos ofrece acontecimientos bien localizados, nos muestra las difíciles y cotidianas situaciones que deben enfrentar un buen número de personas trans para vivir en una sociedad como la nuestra. Identifica instituciones específicas y los requerimientos que demandan para reconocer una identidad como legítima. Ofrece hipótesis e interpreta las circunstancias. Define relaciones y expresa la particular manera en que él mismo se posiciona en este entramado amenazante. En suma, ofrece una mirada donde se contextualiza la experiencia en lugares, tiempos y actores específicos.

Además, en otro vínculo con la acción social, esta metodología reconoce la actividad agenciada del sujeto en la construcción de un relato particular, propio de su posición y visión del mundo. Por su propia naturaleza, la producción de una narrativa exige un trabajo de selección, de ordenamiento y concatenación, lo que implica establecer márgenes, delimitar, incluir o descartar elementos. A través de una narrativa se generan dispositivos de cohesión que permiten hilar acción; las narrativas construyen entramados que sostienen y desarrollan el sentido de la experiencia de las narradoras y su circunstancia. Atendamos lo que nos cuenta Camila, una chica travesti mexicana:

Aquí es peor porque sólo con que un hombre te acuse te tratan como un delincuente (...) El gobierno es de lo peor en cuestiones de los gay y las travestis. Tú como travesti si llamas a la policía porque algo te pasó, te levantan a ti y al criminal o el que te robó o el que te hizo algo, se queda como la víctima y uno como travesti, sólo por el

\footnotetext{
${ }^{3}$ Algunas de las personas participantes utilizaron seudónimo para firmar su narrativa en cuyo caso la narrativa se referencia con el nombre elegido. En el caso de participantes que firmaron sus narrativas con sus nombres reales los fragmentos de narrativa se referencian con la inicial de su nombre seguida del primer apellido.
} 
hecho de serlo, te quedas como el agresor. (Camila, Colima, Narrativa, Agosto de 2010).

En este extracto de la narrativa observamos la denuncia de situaciones y actores específicos y el encuadre de los acontecimientos en contextos físicos y culturales concretos. Puede observarse la vinculación entre planos macrosociales y situaciones cotidianas microsociales. Sus palabras proveen marcos de comprensión sobre las dificultades que enfrentan las personas trans de su contexto y sobre su propia posición de vulnerabilidad, vehiculizando la narración en torno a un particular tema de interés y denuncia para la participante. Esta narración permite, además, hacer evidentes los modos en que se entrelazan diferentes formas de violencia y la forma en que éstas se tejen con la subjetividad de los sujetos que las padecen.

En el plano de la relación metodológica, las producciones narrativas reconocen y promueven la generación de conocimiento como una actividad de colaboración y co-construcción de sentido. Contar historias es una actividad relacional, una práctica colaborativa entre quien cuenta y quien escucha. En este sentido, la producción de narrativas hace emerger, en la situación de investigación, nuevas formas de relación (vinculadas al reconocimiento y al esfuerzo de mutuo entendimiento) donde se movilizan aspectos relacionados con el poder y con la horizontalidad de la relación.

Este abordaje presupone una interacción entre la narrativa del sujeto y el trabajo de la investigadora: al producirla, editarla, analizarla y reportarla. Reconoce, además, un trabajo de la participante en el análisis y edición de su propia narrativa, en la autoridad que mantiene y comparte sobre el discurso en construcción. Finalmente, este abordaje defiende un trabajo colaborativo de creación de relatos con sentido a partir de los intereses y deseos de ambas partes: investigadora y participante.

En su intento por generar una experiencia de género inteligible para otras personas, de comunicar su particular y compleja vivencia en relación con la masculinidad y la feminidad, Camila construye un relato que allana terreno de comprensión común con la investigadora y, más allá, con una comunidad de posibles lectoras:

En mi vida cotidiana no ando vestido de mujer, vestirme de mujer es mi parte de diversión de noche. Decía un amigo que 'ando de civil', pero los fines de semana se me antoja vestirme, agarrar las zapatillas, la peluca, el maquillaje (...) Normalmente ando vestido de hombre porque me gusta la comodidad del pantalón, los zapatos. Vestirte de mujer es realmente bien interesante, es depilarte, 'truquearte' la cara, 'truquearte' todo, ponerte la peluca, las zapatillas, a veces es muy incómodo, pero lo bonito de esto es la atención de la gente, me encanta la atención. (Camila, Colima, Narrativa, Agosto de 2010).

En contraparte, la investigadora recoge y ordena las palabras de la participante, reconoce su peculiar experiencia de género y eventualmente otorga, en conexión con otras narrativas, sentido teórico con respecto a la misma. Las devoluciones y las añadiduras sobre relato abren espacio para el intercambio de comprensiones e intereses, generan una narración que es producto de una actividad dialógica y que, más que reflejar una identidad original del sujeto participante, construye y moviliza una determinada perspectiva sobre el tema en un contexto político y social.

$Y$ aquí reside precisamente un vínculo entre narrativa y acción particularmente sugerente en el análisis crítico del dispositivo de sexogenérico. Este vínculo se refiere a la posibilidad de las narrativas de participar e incidir en discusiones y controversias donde las políticas de género se ponen en juego. Producir narraciones y relatos en contextos determinados y sobre un objeto en disputa, es también una forma de participar en las conversaciones donde se establecen los discursos normativos y los sistemas simbólicos que regulan la experiencia generizada.

En este sentido, la co-producción de narrativas puede ser pensada como un instrumento de investigación y acción en el contexto de las disputas en torno a los "regímenes de verdad" (Foucault, 1988/1990) y a las condiciones de posibilidad de diversas expresiones e identidades sexo-genéricas. Para Michel Foucault, la noción de régimen de verdad se refiere a los juegos de verdad que se establecen en el campo social y de los cuales somos a la vez sujeto y objeto. Se refiere también a las relaciones que establecemos con nosotras mismas y con otras a través de diferentes saberes $y$, particularmente, del conocimiento científico. Así, un régimen de verdad establece relaciones entre conocimiento, poder y sujeto: 
Cada sociedad tiene su régimen de verdad, su "política general de la verdad": es decir, los tipos de discursos que ella acoge y hace funcionar como verdaderos; los mecanismos y las instancias que permiten distinguir los enunciados verdaderos o falsos, la manera de sancionar unos y otros; las técnicas y los procedimientos que son valorizados para la obtención de la verdad; el estatuto de aquellos encargados de decir qué es lo que funciona como verdadero (Foucualt, 1988/1990, p. 187).

Otra forma de reconocer este carácter activo de las narrativas en el contexto de discusiones sobre las verdades del género se encuentra en su función argumentativa o retórica ya advertida por Michael Billig (1991). Los textos y los relatos, incluyendo aquellos que son producto de la colaboración metodológica entre participante e investigadora, se organizan y se movilizan en función de interacciones dialógicas. En este sentido, las narrativas y explicaciones en torno a las controversias de sexo/género son siempre movimientos persuasivos y deliberativos, participan en polémicas donde se van construyendo las categorías y las relaciones que habitamos. Las narraciones actúan en un contexto argumentativo, se movilizan en el terreno público, a favor y en contra de determinadas posibilidades.

Consideremos la forma en que Pau, activista trans, constituye una narrativa personal en oposición a la perspectiva médico-psiquiátrica dominante sobre las identidades transgénero:

¿Cuál es mi relación o vinculación con el trastorno de la identidad de género? Al nivel de mi autopercepción, ninguna. No creo tener ningún tipo de trastorno, no me considero un "enfermo mental". Sin embargo, está claro que en la práctica cotidiana la noción de este trastorno me afecta porque, por ejemplo, necesito el visto bueno de un psiquiatra para poder acceder a un cambio legal de sexo y nombre en los documentos oficiales. Pero la intrusión no es solo a nivel administrativo: es posible palpar cotidianamente cómo la figura del experto pretende hablar por mí y, por otro lado, cómo la presión social puede ser tan fuerte que puede hacer que la diferencia se experimente como una enfermedad (P. Crego Walters, Barcelona, Narrativa, Febrero de 2008).

En las palabras de Pau podemos encontrar un desafío al régimen discursivo patologizante, la expresión de una comprensión sobre su propia identidad, al mismo tiempo que un reconocimiento de los poderosos constreñimientos a los que se ven sujetadas las personas transgénero a través del sistema dominante sexo/género. De esta manera, como dispositivos culturales y psicosociales, las narrativas alinean fuerzas y elementos disímiles en con- figuraciones productivas de diferencia y oposición. A menudo, las historias situadas reflejan modos de transgresión y ruptura con respecto a los discursos dominantes de gran complejidad, atravesados por discursos institucionales disímiles y experiencias personales frecuentemente invisibilizadas.

Finalmente, puede entenderse que la producción de narrativas es una acción social en el sentido de que, en el contexto de una discusión, permite precisamente tomar la palabra. A través de esta "toma de palabra" la narradora se constituye como sujeto de enunciación, da cuenta de su propia posición y perspectiva, esto es, participa en los juegos dialógicos donde se construyen nuestras formas de vida $y$, entre otras cosas, lo que asumimos como verdad y conocimiento.

Para Paolo Virno (2005, p. 67), tomar la palabra constituye "el presupuesto oculto de todos los habituales enunciados performativos, eso que les permite realizar una u otra acción particular". Tomar la palabra, narrar, producir discurso, es también actuar en el sentido de que implica asumir una postura, construir un lugar en un entramado de relaciones. Como ha hecho notar Teresa de Lauretis (1984), el argumento también es una confrontación, una lucha, una intervención política en instituciones y en la práctica de la vida diaria. El hecho de que la confrontación tenga un carácter discursivo -en el sentido de que el lenguaje y las narraciones están siempre incrustados en las prácticas de la vida cotidiana, donde al final reside el significado- está implícito en una de las proposiciones clave del feminismo: lo personal es político.

Las palabras de Nancy, activista transgénero en Colima, pueden mostrarnos este gesto de tomar la palabra para definirse a sí misma:

\begin{abstract}
Me considero una persona transgénero. Estoy a la mitad, en el proceso de en medio entre un sexo y el otro. Hay hombres que se visten de mujer pero que no utilizan hormonas. Yo sí utilizo hormonas, pero no es mi deseo ser completamente transexual. No quiero cambiar de sexo (operarme los genitales) ni operarme las bubis. No me interesa llegar a ese a nivel, me basta con lo que me sale de las hormonas, no necesito implantes ni más modificaciones. Por eso me considero transgénero, porque estoy en medio de los dos sexos (Nancy, Colima, Narrativa, Noviembre de 2010).
\end{abstract}

En esta narración se muestra un acto de autodefinición y afirmación de la propia identidad - una identidad que no cabe en los cánones 
binarios de las meta-narrativas dominantes de género- que se realiza desde una vivencia singular del propio cuerpo. En contraposición a la idea de "dar voz", frecuente en la investigación social sobre identidades subalternizadas, la producción de narrativas permite abrir un espacio para tomar la palabra, para generar comprensiones afirmativas, en primera persona y posicionadas en un campo de conocimiento.

En suma, comprender las narrativas no sólo como instrumentos de investigación sino también como vehículos para la acción permite expandir sus alcances teórico-metodológicos y concebir su funcionamiento en al menos dos planos simultáneos: a) como un abordaje que busca acceder a la subjetividad, a la posición de un sujeto que da sentido a sus experiencias y da cuenta del contexto social en que se desenvuelve; y b) como abordaje de la performance discursiva, como un espacio de interacción discursiva que genera ciertas posiciones de sujeto. Así, la producción de narrativas como instrumento metodológico permite transitar una vía en dos direcciones: el actor construye un relato y el relato genera una posición identitaria.

Las narrativas co-producidas pueden entenderse como "artefactos", como aparatos semióticos y materiales que pueden ser movilizados en diferentes espacios con objetivos de transformación social y política (Johnson y Martínez-Guzmán, 2013). Estas narraciones pueden representar recursos útiles, por ejemplo, para informar la reflexión sobre métodos de investigación, para ampliar la comprensión de la salud mental, para informar procesos activistas, para visibilizar violencias soterradas, para complejizar la comprensión de las masculinidades y feminidades en el contexto académico/educativo $y$, en última instancia, para ofrecer testimonios críticos en la lucha por transformar esquemas de género coercitivos y excluyentes con respecto a las identidades de género no normativas.

\section{Nuevos relatos}

El argumento desarrollado en este artículo ha buscado mostrar la potencialidad de la perspectiva narrativa en el estudio del se$\mathrm{xo} /$ género $\mathrm{y}$, particularmente, de las identidades no normativas. El abordaje narrativo permite generar una perspectiva situada y localizada del fenómeno de estudio, a partir de la subjetividad encarnada de la narradora y de elementos indexicales del relato. Además, la narrativa reconoce el lugar de un sujeto con agencia y creatividad, da cuenta del trabajo identitario que realiza la participante y de la producción agenciada de su propio relato.

La aproximación narrativa permite, asimismo, acceder de manera privilegiada a tramas donde lo micro y lo macro social, lo personal y lo político, se entrelazan, ofreciendo así una perspectiva integradora y compleja de las relaciones de género. Asimismo, el abordaje narrativo facilita poner en relieve el trabajo dialógico y colaborativo que se desarrolla en el encuentro metodológico y que genera un conocimiento de naturaleza relacional.

Hemos buscado mostrar, además, la forma en que el abordaje narrativo de investigación puede concebirse como una herramienta metodológica no sólo de producción de conocimiento sino también de acción social -en la medida de que la producción de conocimiento es también un acción social- en contextos donde se discuten y se definen los entramados discursivos que sostienen determinadas relaciones de género. Para enfatizar y potenciar este carácter activo de la investigación narrativa, se ha discutido la riqueza y la utilidad de la propuesta metodológica de las producciones narrativas (Balasch y Montenegro, 2003).

El modo principal en que las producciones narrativas constituyen una herramienta de investigación y de acción en el ámbito del género se vincula precisamente con la elaboración de nuevos relatos en colaboración con las participantes. Estos nuevos relatos permiten visibilizar posiciones situadas y vivencias sexo-genéricas disruptivas que con frecuencia permanecen en el silencio porque desafían, en alguna medida, las meta-narrativas dominantes sobre el sexo/género. Estos relatos permiten, además, multiplicar las formas posibles de narrar y comprender las identidades y las relaciones sexo-genéricas.

Comprender la forma en que se estructuran y se movilizan las narraciones en contextos determinados permite cuestionar y empujar los límites de la envoltura de la identidad, permite identificar $y$, por tanto, analizar críticamente las formas canónicas establecidas para adquirir credibilidad como narradora de 
la verdad: "contar la verdad es una actividad que se encuentra ampliamente regida por una preestructura de convenciones narrativas" (Gergen, 2007, p. 158). Más aún, las narrativas pueden tener diferentes valencias políticas y epistemológicas en función de los contextos donde son producidas y leídas. Así, es posible identificar las condiciones a partir de las cuales se producen narrativas hegemónicas -relatos que reproducen y sostienen las relaciones de poder y desigualdad establecidas- y promover la elaboración de historias 'subversivas' - narrativas que desafían los presupuestos hegemónicos a través de la explicitación de las conexiones entre trayectorias de vida singulares y la organización general de la vida social- (Ewick y Silbey, 1995).

Cuando los grandes relatos (culturales o científicos) son cuestionados y pierden su halo de universalidad y trascendencia, se abre la posibilidad a la emergencia de múltiples relatos más cotidianos, contextualizados y diversos entre sí, que es posible articular para generar nuevos sentidos. En este caso, la función de la investigadora bien puede ser, como lo ha propuesto Norman Denzin (1991, p. 156), la de "contar historias". En este contexto, un nuevo relato no es sólo una variante de una historia ya conocida, sino un dispositivo que permite refutar la hipótesis de que la historia había llegado a su fin, que no podía ser contada de otra manera. La elaboración de nuevos y diferentes relatos permite traer a la luz que ninguna historia $y$, ciertamente, ninguna forma de leer o interpretar una historia, es definitiva e inevitable.

\section{Referencias}

Ankersmit, Frank R. (2001). Historical Representation. Stanford: Stanford University Press.

Balasch, Marcel, y Montenegro, Marisela (2003). Una propuesta metodológica desde la epistemología de los conocimientos situados: Las producciones narrativas. Encuentros en Psicología Social, 1(3), 44-48.

Barthes, Roland (1977). Image, Music, Text. Nueva York: The Noon Day Press.

Bauer, Marin (1996). The narrative interview: comments on a technique of qualitative data collection (LSE Methodology Institute Papers Qualitative series, 1). Disponible en: http://www.lse.ac.uk/methodology/pdf/QualPa pers/Bauer-NARRAT1SS.pdf
Billig, Michael (1991). Arguing and thinking: a rhetorical approach to social psychology. New York: Cambridge University Press.

Blumenfeld-Jones, Donald (1995). Fidelity as a criterion for practicing and evaluating narrative inquiry. En J. Amos Hatch y Richard Wisniewski (Eds.), Life History and Narrative (pp. 25-35). London: The Falmer Press.

Boje, David M. (1991). The storytelling organization: A study of story performance in an officesupply firm. Administrative Science Quarterly, 36, 106-126. http://dx.doi.org/10.1177/105649269984002

Bruner, Jerome (1991). The narrative construction of reality. Critical Inquiry, 18(1), 1-21.

Bruner, Jerome (1986). Actual minds, possible worlds. Cambridge: Harvard University Press.

Butler, Judith. (1999/2007). El Género en Disputa. El Feminismo y la Subversión de la identidad. Barcelona: Paidós.

Clandinin, Dean J. y Connelly, F. Michael (1994). Personal experience methods. En Norman K. Denzin e Yvonna S. Lincoln (Eds.), Handbook of qualitative research (pp. 413-427). London: Sage.

Clegg, Stewart R. y Bailey, James (Eds.) (2008) International Encyclopaedia of Organization Studies. Thousand Oaks, CA: Sage.

Clough, Peter (2002). Narratives and Fictions in Educational Research. Buckingham: Open University Press.

Cole, Barbara (2009). Gender, Narratives and Intersectionality: can Personal Experience Approaches to Research Contribute to "Undoing Gender"? International Review of Education, 55(5/6), 561-578. http://dx.doi.org/10.1007/s11159-009-9140-5

Crossley, Michele L. (2000). Introducing narrative psychology: self, trauma, and the construction of meaning. Buckingham: Open University Press.

De Lauretis, Teresa (1984). Alice Doesn't: Feminism, Semiotics, Cinema. Bloomington: Indiana University Press.

Denzin, Norman (1991). Images of posmodernism. London: Sage.

Ewick, Patricia y Silbey, Susan S. (1995). Subversive stories and hegemonic tales: Toward a sociology of narrative. Law \& Society, 29(2), 197226.

Foucault, Michel (1988/1990). Tecnologías del yo y otros textos afines. Barcelona: Paidós. 
Freeman, Elizabeth (2010). Time Binds: Queer Temporalities, Queer Histories. Durham: Duke UP.

Gergen, Kenneth J. (2007). Construccionismo social, aportes para el debate y la práctica. Bogotá: Universidad de Los Andes.

Haraway, Donna (1995). Ciencia, cyborgs y mujeres. La reinvención de la naturaleza. Madrid: Cátedra.

Harding, Sandra (1996). Ciencia y Feminismo. Madrid: Ediciones Morata.

Hatch, J. Amos y Wisniewski, Richard (Eds.) (1995). Life History and Narrative. London: The Falmer Press.

Íñiguez, Lupicinio (Ed.) (2003). Análisis del Discurso. Manual para las Ciencias Sociales. Barcelona: UOC.

Johnson, Katherine y Martínez-Guzmán, Antar (2013). Rethinking concepts in Participatory Action Research and their potential for social transformation: Post-structuralist informed methodological reflections from LGBT and TransCollective projects. Journal of Community \& Applied Social Psychology, 23, 1-15. http://dx.doi.org/10.1002/casp.2134

Kreiswirth, Martin (1992). Trusting the Tale: The Narrativist Turn in the Human Sciences. New Literary History, 23, 629-657.

Lanser, Susan S. (1986). Toward a Feminist Narratology. Style, 20(3), 342-363.

Lanser, Susan S. (2013). Revision of Gender and Narrative. The living handbook of naratology. Disponible en: http://www.lhn.unihamburg.de/node/86/revisions/116/view

Lyotard, Jean-François (1984). La condición postmoderna. Madrid: Cátedra.

Madison, D. Soyini (1998). Performances, Personal Narratives, and the Politics of Possibility. En Sheron J. Dailey (Ed.), The Future of Performance Studies: Visions and Revisions (pp. 276 286). Annadale, VA: National Communication Association.

Martínez-Guzmán, Antar e Íñiguez-Rueda, Lupicinio (2010). La fabricación del Trastorno de Identidad
Sexual: Estrategias discursivas en la patologización de la transexualidad. Discurso \& Sociedad, 4(1), 30-51.

Peterson, Amy (2006). An African-American woman with disabilities: the intersection of gender, race and disability. Disability and Society, 21(7), 721734.

http://dx.doi.org/10.1080/09687590600995345

Pinnegar, Stefinee y Daynes, Gary (2007). Locating narrative inquiry historically: Thematics in the turn to narrative. En Jean Clandinin, (Ed.), Handbook of narrative inquiry: Mapping a methodology (pp. 1-34). Thousand Oaks, CA: Sage.

Polkinghorne, Donald (1988). Narrative knowing in the human sciences. Albany: State University of New York Press.

Potter, Jonathan y Wetherell, Margaret. (1996). El Análisis del Discurso y la Identificación de los Repertorios Interpretativos. En Gordo, Angel y Linaza, José Luis (Eds.), Psicologías, Discursos y Poder (PDP) (pp. 63-78). España: Visor.

Ricoeur, Paul (2000). Narratividad, fenomenología y hermenéutica. Anàlisi, 25, 189-207.

Riessman, Catherine K. (2002). Analysis of personal narratives. En Jaber F. Gubrium y James A. Holstein (Eds.), Handbook of Interview Research (pp. 695-710). Thousand Oaks: Sage.

Riessman, Catherine K. (2008). Narrative methods for the human sciences. California: Sage.

Roof, Judith (1996). Come As You Are: Sexuality and Narrative. New York: Columbia University Press.

Rorty, Richard (1998). El giro lingüístico. Barcelona: Paidós / ICE-UAB.

van Dijk, Teun A. (Ed.) (1997). Discourse studies. London: Sage.

Virno, Paolo (2005). Cuando el verbo se hace carne. Lenguaje y naturaleza humana. Madrid: Traficantes de sueños.

Wodak, Ruth (2003). ¿De qué trata el análisis crítico del discurso (ACD)? En Ruth Wodak y Michael Meyer (Eds.), Métodos de análisis crítico del discurso (pp. 17-35). Barcelona: Gedisa. 


\section{ANTAR MARTÍNEZ-GUZMÁN}

Doctor en Psicología Social por la Universidad Autónoma de Barcelona (UAB) y miembro del grupo "Fractalidades en Investigación Crítica". Sus líneas de trabajo giran en torno a los procesos identitarios y las corporeidades en el ámbito de sexo-género, el análisis crítico de los discursos científicos clínicos y la reflexión sobre tecnologías de investigación-acción psicosocial. Profesor de la Facultad de Psicología de la Universidad de Colima.

\section{MARISELA MONTENEGRO}

Profesora del Departamento de Psicología Social y miembro del grupo "Fractalidades en Investigación Crtítica". Co-coordinadora del Máster de Investigación e Intervención Psicosocial de la Facultad de Psicología de la UAB. Ha analizado aspectos epistemológicos, metodológicos, políticos y éticos de la investigación e intervención social y las maneras de producción y transformación social, analizando fenómenos -servicios para personas inmigradas, movimientos sociales o nuevas tecnologías- desde una perspectiva crítica.

\section{DIRECCIÓN DE CONTACTO}

antar_martinez@ucol.mx

\section{FORMATO DE CITACIÓN}

Martínez-Guzmán, Antar y Montenegro, Marisela (2014). La producción de narrativas como herramienta de investigación y acción sobre el dispositivo de sexo/género: Construyendo nuevos relatos. Quaderns de Psicologia, 16(1), 111-125. http://dx.doi.org/10.5565/rev/qpsicologia.1206

\section{HISTORIA EDITORIAL}

Recibido: 28/03/2014

$1^{a}$ Revisión: 12/05/2014

Aceptado: 20/05/2014 